\title{
Mesenchymal stem cell therapy for cutaneous radiation syndrome
}

\section{Sadanori Akita ${ }^{\text {* }}$, Kozo Akino², Akiyoshi Hirano ${ }^{1}$, Akira Ohtsuru ${ }^{3}$, Shunichi Yamashita 4,5}

${ }^{1}$ Department of Plastic and Reconstructive Surgery, ${ }^{2}$ Department of Neuroanatomy, Nagasaki University, Graduate School of Biomedical Sciences, ${ }^{3}$ Takashi Nagai Memorial International Hibakusha Medical Center, Nagasaki University Hospital, ${ }^{4}$ Atomic Bomb Disease Institute, Nagasaki University School of Medicine, ${ }^{5}$ World Health Organization

Correspondence person: Sadanori Akita 1-7-1 Sakamoto, Nagasaki 852-8501, Japan.

e-mail: akitas@hf.rim.or.jp

grant: Nagasaki University Global COE Program, "Global Strategic Center for Radiation Health Risk Control," by Japan Society for the Promotion of Science. 


\begin{abstract}
Increasing concern on systemic and local radiation injuries caused by nuclear power reactor accident, therapeutic irradiation or nuclear terrorism should be treated and prevented properly for life-saving and improvement of wound management.
\end{abstract}

Recently, regenerative surgical modalities should be attempted with temporal artificial dermis impregnated and sprayed with local angiogenic factor such as basic fibroblast growth factor and secondary reconstruction can be a candidate for demarcation and saving the donor morbidity.

Human mesenchymal stem cells and adipose-derived stem cells, together with angiogenic and mitogenic factor of basic fibroblast growth factor and an artificial dermis were applied over the excised irradiated skin defect are tested for differentiation and local stimulation effects in the radiation-exposed wounds. The perforator flap and artificial dermal template with growth factor were successful for reconstruction in patients who are suffering from complex underlying disease. Patients were uneventfully treated with minimal morbidities.

In the experiments, the hMSCs are strongly proliferative even after 20Gy irradiation in vitro. In vivo, 4Gy rat whole body irradiation demonstrated the sustained marrow stromal (mesenchymal stem) cells survived in the bone marrow. Immediate artificial dermis application impregnated with cells and the cytokine over the 20 Gy irradiated skin and soft tissues demonstrated the significantly improved fat angiogenesis, architected dermal reconstitution and less inflammatory epidermal recovery.

Detailed understanding of underlying diseases and rational reconstructive procedures brings about good outcomes for difficult irradiated wound healing. Adipose-derived stem cells are also implicated in the limited local injuries for short cell harvesting and processing time in the same subject.

\title{
KEYWORDS: $\quad$ exposure, Nagasaki , radiation damage, radiotherapy
}




\section{INTRODUCTION}

There is increasing worried on both systemic and local radiation injuries probably caused by nuclear power plant (NPP) reactor accidents, therapeutic irradiation for malignancy, interventional radiology (IRV) of unexpectedly prolonged fluoroscopic procedures for cardiovascular diseases such as arrhythmia, ischemic heart diseases or nuclear medicine of over-dose intake of the radioactive for nuclear medicine of internal radiation therapy. These conditions should be treated and prevented properly for sake of life-saving and improvement of local wound healing (Francois, Mouiseddine, Mathieu, Monti, Dudoiqnon, Sache, Boutarfa, Thierry, Gourmelon, Chapel 2007). However, total clinical analysis and experimentally evidence-based data were not founded yet. Nagasaki University of authors' group is selected as global strategic center for radiation health risk control by the Japan's Ministry of Education, Culture, Sports and Technology and now exploring to establish therapeutic regimens, prevention the radiation injuries and possible regeneration medical and surgical therapy for radiation injuries by using patients' own adipose tissue-derived stem cell therapy.

Often seen chronic radiation injuries are well handled by sufficient enough blood supply to the radiated tissues, especially in the cartilage, bare bone, and hardened scar tissues. For this purpose, local-, distant- and microsurgical vascularized flap are applied. Recent development of micro-vasculature of the skin and soft tissues including the connective tissues plays major roles in attributing to accelerate local wound healing. Also, externally administered angiogenic growth factor such as basic fibroblast growth factor (bFGF) together with temporal wound coverage of artificial skin substitute is very effective for those patients with severely injured patients with co-morbidities, who are intolerant to the extensive and long surgeries (Akita, Tanaka, Hirano 2006). In contrast, acute phase radiation injury demonstrates often fluctuated response to the medication and surgery. Also, systemic exposure of radiation often ameliorates the body immune response, cellular proliferation, differentiation capacity in total body, thus early administration of cells, preferably radiation-resistant, cell-renewal and of high differentiation capacity, in this context, stem cells from the bone marrows or 
adipose cells are recommended. In order to elucidate efficacy of these stem cells, both in vitro and in vivo experiments are undertaken.

\section{MAERIALS AND METHODS}

\section{Chronic local radiation injuries}

Often experienced in radiation therapy for malignancy, cardiovascular modalities, these should be categorized as difficult wounding with poor vasculature.

From January 1990 to April 2007, 10 (8 females and 2 male) patients who demonstrated radiation injuries such as telangiectasia, xerosis, epidermal atrophy karatoses, and fibrosis as well as deep ulcers in the costal ribs and sternum by adjuvant radiation therapy post-mastectomy and prolonged fluoroscopic procedures for cardiovascular diseases, were surgically treated and included in this investigation.

Other selective clinical cases were used angiogenic growth factor namely human recombinant basic fibroblast growth factor ( $\mathrm{rh}-\mathrm{bFGF}$ ), which is clinically approved and widely used for clinical wounds in Japan with skin substitutes, which are also clinically available not only in Japan but many other nations including USA, majority of EU nations and several Asian counties, and the effectiveness of using the artificial skin substitutes in the chronic radiation injuries is temporal coverage and sustainability of both internal and external cells and growth factors. Therefore, combined use of bFGF and artificial skin substitute leads to improved quality of wounds (scars) as well as facilitated wound healing (Akita, Akino, Tanaka, Anraku, Hirano 2008). Additionally, one case was treated with autogenous adipose-derived stem cell (ADSC) for sacral radiation ulcer for the first time in the world, which was caused by a therapeutic radiation at fractionate 50 Gy at 40 years previously.

\section{Acute local radiation injuries}


When the radiation does not affect harvesting donor-sites such as abdomen, thighs, buttock and arms, ADSC, or adipose-derived regenerative cells (ADRC) are often of choice of immediate regeneration for radiation-exposed wounds, since the lipoaspirated fat cells are easily processed within a few hours in a closed circuit of the processing machine only used for each specific patient. Internal Review Board (IRB) of ethic committee of Nagasaki University approved us this modality for the radiation injured wound healing (No. 08070296).

\section{Acute systemic radiation injuries}

Extensive both in vitro and in vivo studies are explored using human mesenchymal stem cells, since these cells are readily available in frozen cell stockpiles and thus will be potential therapeutic regimens for unscheduled radiation injuries. Also, the adipose-derived stem cells are also the candidate for stem cell banking and stockpile.

\section{An in vivo model and whole body irradiation by an X-ray generator}

Animals were aged 10 weeks and weighing 300-350 g, and were used. Animals were obtained from CLEA JAPAN (Tokyo, Japan), housed in the laboratory animal centre for biomedical research, Nagasaki University School of Medicine (Nagasaki, Japan), and the protocol of the animal experiment was approved by the Institutional Animal Care and Use Committee of Nagasaki University, no. 0204080111. They were handled according to the guidelines established for animal care at the centre. Each rat had free access to both sterile water and standard rodent soft chow ad libitum.

4 Gy or 20 Gy whole body irradiation to 10 nude rats (F344/NJCl-rnu), which are deleted T-cell function and thus acute immune rejection to human derived cells is minimized, were performed at Atomic bomb Disease Institute, Nagasaki University, by a X-ray radiation generator (EXS-300-5, Toshiba, 200kV, $15 \mathrm{~mA}$, $0.405 \mathrm{~Gy} / \mathrm{min}$ ). Animals were divided into two groups of five each, control group and $\mathrm{hMSCs}^{-}$with bFGF-treated group and surgical procedures were performed immediately after irradiation.

\section{Angiogenic growth factor, basic fibroblast growth factor (bFGF)}


Genetically recombinant human bFGF (Fiblast ${ }$, Trafermin) was purchased from Kaken Pharmaceutical Co., Inc (Tokyo, Japan). The freeze-dried samples were dissolved in PBS at a concentration of $1 \mathrm{mg} / \mathrm{mL}$ and dissolved in culture medium 30 min before experimental use.

\section{RESULTS}

\section{Chronic local radiation injuries}

All surgeries were uneventfully performed with the mean postoperative followup was 11 years and 3 months (3 year to 16 years) and the average age was 67 years (53 to 78 years). Above all, in the cases of the compromised hosts such as aged with systemic conditions, there is already successfully treated with the patient own adipose-derived stem cells for the intractable local radiation injury in our institute. The patient is very old and first eliminated for this new modality, however, considering the patient's other condition, this clinical trial was applied to and in 81day after regenerative surgery the chronic radiation injury completely healed and demonstrated the durable for the external stimuli in three weeks at 103 days post-operatively (Fig. 1 ).

\section{Acute local radiation injuries}

Autologous adipose derived stem cells (or "regenerative" cells), which are distant from radiation sites, are most favourable for regeneration source and conditioning for the pre-reconstruction procedures. When the radiation safely enough distant from the adipose cell donor sites such as lower abdomens, tights, buttocks and arms, then liposuction of the adipose cells are started. Approval for treatment for the radiation injuries is now obtained through IRB of Nagasaki University and the adipose cell processing in the operation room within 2 hours is underway using a Celution ${ }^{\mathrm{TM}}$ system with a collaboration of Cytori Therapeutics, Inc. (San Diego, USA). Preliminary study of the cell characteristics of the ADSC, or ADRC are very comparable to those of the hMSCs and thus experimental data with hMSCs may be applicable to the ADSC/ADRC in 
regeneration and wound healing. Among notable characteristics, multi-lineage differentiation mechanisms promote the complex difficult wound healing in regards to epithelialisation, neo-vascularization and matrix deposition by fibroblast production (Zuk, Zhu, Ashjian, De Ugarte, Huang, Mizuno, Alfonso, Fraser, Benhaim, Hedrick 2002).

\section{Acute systemic radiation injuries}

\section{An in vitro stem cell biology and analysis}

In order to investigate human mesenchymal stem cell proliferation, subconfluent cultured hMSCs were used and irradiated by an X-ray radiation generator. The cells were immediately transferred to the incubators after irradiation. For control cells of different species of origins are used. Both human neuroblastoma cellls (NG1087-15) and rat pheochromocytoma cells (PC-12) are used. Cell proliferation was consistent in three cell groups in the normal condition (no radiation and normal medium), however, 20Gy irradiation caused cell death in groups of NG1087-15 and PC-12 in 48 hours, in contrast, the hMSCs survived up to next 96 hours.

In an electron microscopy, irradiated hMSCs demonstrated surface microvilli all over the cells, however the hMSCs still survived after 60 Gy irradiation, of which dose is considered medium and induce the significant intestinal bleeding.

\section{An in vivo analysis after whole body irradiation}

After 20Gy whole body irradiation, the seemingly radiation-affected surfaces were removed surgically including the panniculus carnosus. Immediate resurfacing with skin substitutes impregnated with hMSCs and bFGF facilitated wound healing. At day 10, the histology demonstrated the vascular rich subcutaneous tissues with more interstitial cellularity.

In lower dose, 4Gy, irradiation to the whole rat body and bone marrow histology demonstrated loss of the hematopoietic lineage cell but marrow stromal cells were survived (Fig. 2) 


\section{Discussion}

Management of radiation injuries compose two major parts. One is localized injuries and the other is of systemic injuries. Among localized radiation injuries, chronic injuries are more common in the medical field after caner radiation therapy. Usually management of these chronic wounds are well-handled by wellvascularized tissue transfers as various plastic surgical procedures have proved. In consideration of each patient general condition and preference, the choice of therapeutic selections should be performed. On the other hand, when the local radiation injuries are encountered in an acute phase, there are high chances for innovative procedures using autogenous stem cells. Since hMSCs are resistant to radiation as demonstrated in vitro cell proliferation curve and also able to produce protein avoiding cell apoptosis (Chen, Lin, Chen, Yang, Chen, Liao, Liu, $\mathrm{Lu}$, Lee 2006). Also, increasing evidences demonstrate that ADSCs are similar to hMSCs in cell properties and characteristics both in vitro and in vivo (Zuk et al. 2002). When localized radiation was distant enough from the donor sites adipose tissues, immediate debridement and regeneration using adipose-derived stem cells, which are available for processing within 1 hours in an simultaneously in a same operation theater without cell culture since adipose tissues (fat tissues) are abundant in adult humans compared to other stem cell sources. In the limited clinical circumstances of high risk patients such as elderly, chronic local infection, there are still opportunity of harvesting and processing the patient-own fatderived stem cells successfully as seen in our case.

For treatment for systemic radiation injuries, stockpiled stem cells should be globally available through medical assistance network system under WHOREMPAN, in which Nagasaki University is highly involved in its activity, or other international frameworks. Early resurfacing of the damaged skin and subcutaneous tissues are as important as hematological and intestinal system resuscitation (Weinstock, Case, Bader, Chao, Coleman, Hatchett, Weisdorf, Confer 2008).

Also, therapeutic guidelines for systemic radiation injuries are anticipated from practical and regulatory view points. Highlighting innovative technology and 
devices such as well as currently existing medicines and devices are expected on behalf of preparing to treat "systemic" radiation injuries most effectively.

Therapeutic regimens of radiation injuries used to be dependent on each subspecialty in the medical filed such as internal medicine, radiology and surgery.

Recent establishment of wound care specialty, mostly led by plastic surgeons but other supporting specialists such as nurses, dermatologists and gastrointestinal physicians and surgeons, may be practically handle these rare but of significant impact "radiation injuries" as a inter-disciplinary approaches (Gottrup 2004). For further more specialized for "radiation injuries" may be required. 


\section{References}

Akita S, Tanaka K, Hirano A. Lower extremity reconstruction after necrotizing fasciitis and necrotic skin lesions using a porcine-derived skin substitute. J Plast Reconstr Aesthet Surg 59: 759-763; 2006.

Akita S, Akino K, Tanaka K, Anraku K, Hirano A. A basic fibroblast growth factor improves lower extremity wound healing with a porcine-derived skin substitute. J Trauma 64: 809-815; 2008.

Chen MF, Lin CT, Chen WC, Yang CT, Chen CC, Liao SK, Liu JM, Lu CH, Lee KD. The sensitivity of human mesenchymal stem cells to ionizing radiation. Int J Radiat Oncol Biol Phys 66: 244-253 ; 2006.

Francois S, Mouiseddine M, Mathieu N, Monti P, Dudoiqnon N, Sache A, Boutarfa A, Thierry D, Gourmelon P, Chapel A. Human mesenchymal stem cells favour healing of the cutaneous radiation syndrome in a xenogenic transplasnt model. Ann Hematol 86: 1-8; 2007.

Gottrup F. A specialized wound-healing center concept: importance of a multidisciplinary department structure and surgical treatment facilities in the treatment of chronic wounds. Am J Surg 187: 38S-43S; 2004.

Weinstock DM, Case C Jr, Bader JL, Chao NJ, Coleman CN, Hatchett RJ, Weisdorf DJ, Confer DL. Radiologic and nuclear events: contingency planning for haematologists/oncologists. Blood 111: 5440-5445; 2008.

Zuk PA, Zhu M, Ashjian P, De Ugarte DA, Huang JI, Mizuno H, Alfonso ZC, Fraser JK, Benhaim P, Hedrick MH. Human adipose tissue is a source of multipotent stem cells. Mol Biol Cell 13: 4279-4295; 2002. 


\section{Figure legends}

Figure 1: World first adipose-derived stem cell therapy for radiation injury

a: Pre-op. The arrow indicates the necrotized exposed sacral bone

b: At 103 days after the ADSC transplantation. Skin, subcutaneous tissue, bone and muscle were regenerated and healed.

c: Harvesting the fat tissue through $5-\mathrm{mm}$ incision by cannulization

$\mathrm{d}:$ ADSC in the syringe. $3.8 \times 10^{7}$ cells were obtained

Figure 2:.Electron microscopy of 4Gy rat whole body irradiated bone marrow

a: Lower magnitude. The hematopoietic cells turned round as seen in white. There are some empty cell shelves demonstrated as black round morphology $(\times 1,500)$.

b: There are predominantly of euchromatin marrow stromal cells. These cells represent the marrow stromal (or mesenchymal stem) cells $(\times 3,000)$.

c: There are some phagocytic macrophages observed $(\times 3,000)$.

Figure 3: Flow chart of therapeutic management of radiation injuries.

Each patient condition should be carefully monitored first. In systemic radiation injuries, after taking in account of triage with consideration of the patients' general conditions and medical environment, stem cell therapy, mainly from the "stockpile" or "stem cell banking" initiated with cytokine augmentation, while local injures are sub-divided into "acute" and "chronic". For less invasive 
therapeutic modality, adipose-derived stem cells are highly recommended even for "severely-injured" or "host-compromised" patients. Each sub-divided group can be excellently handles by experts. 
Fig. 1

a

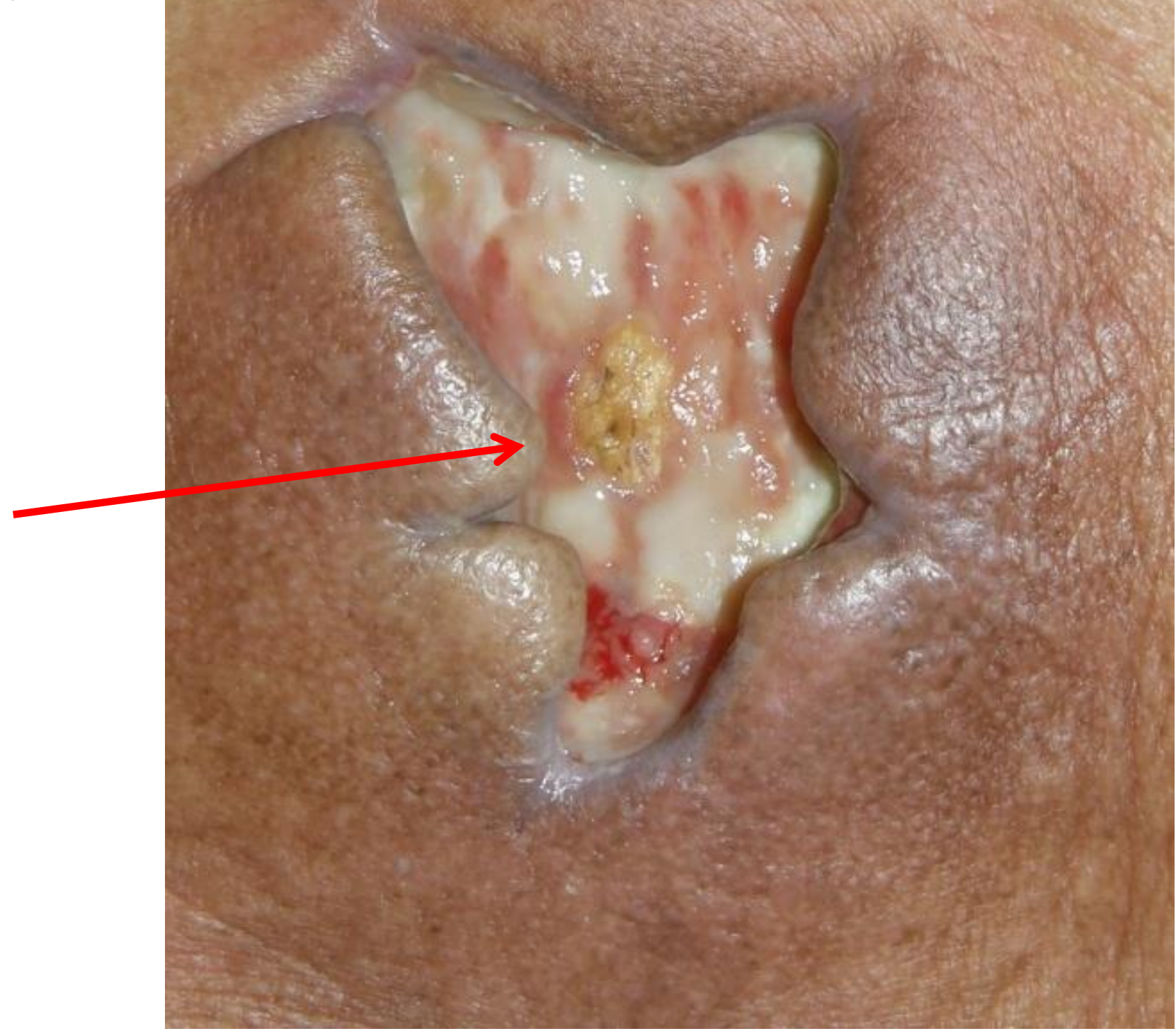


Fig. 1

b

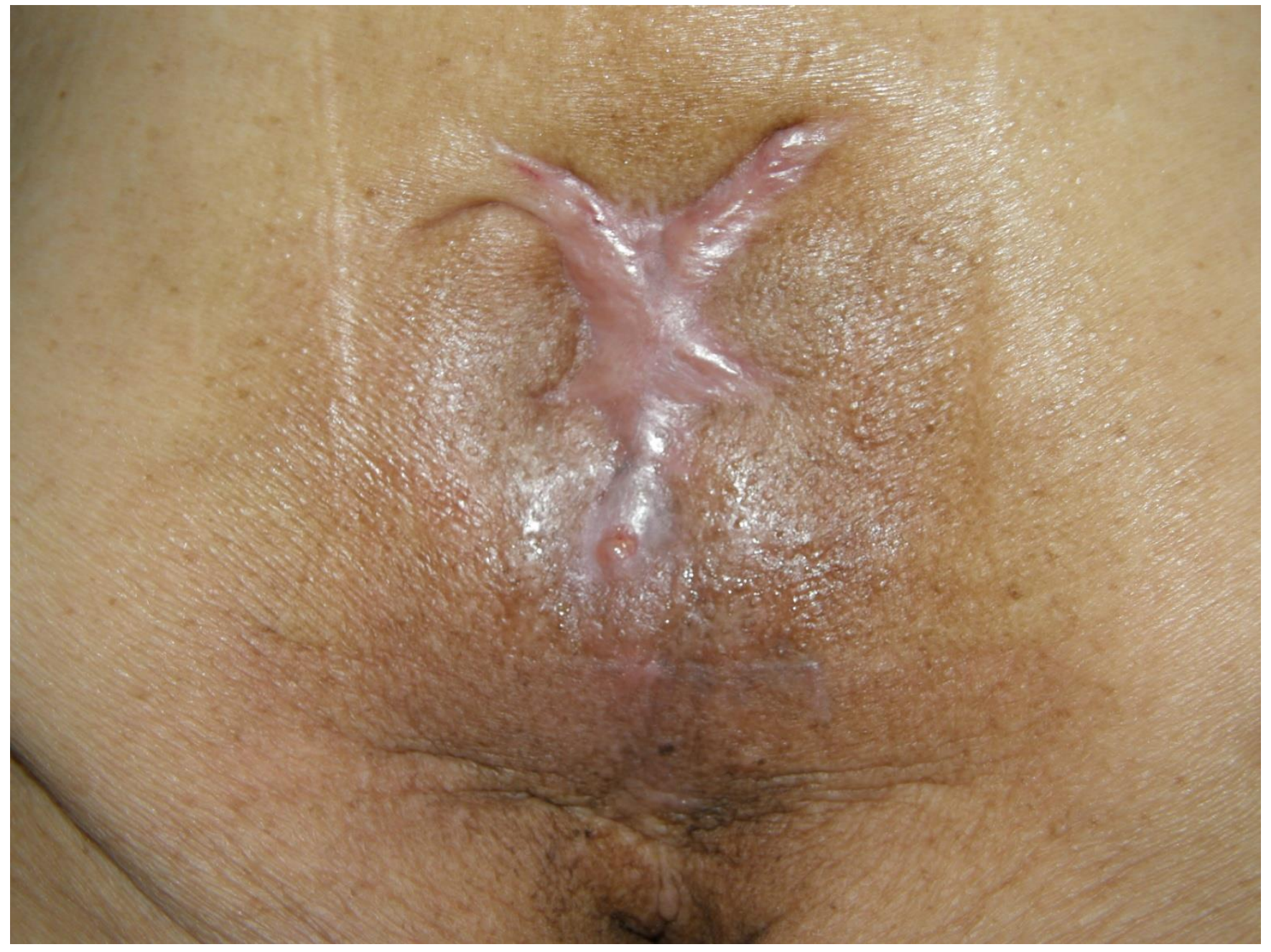


Fig. 1

C

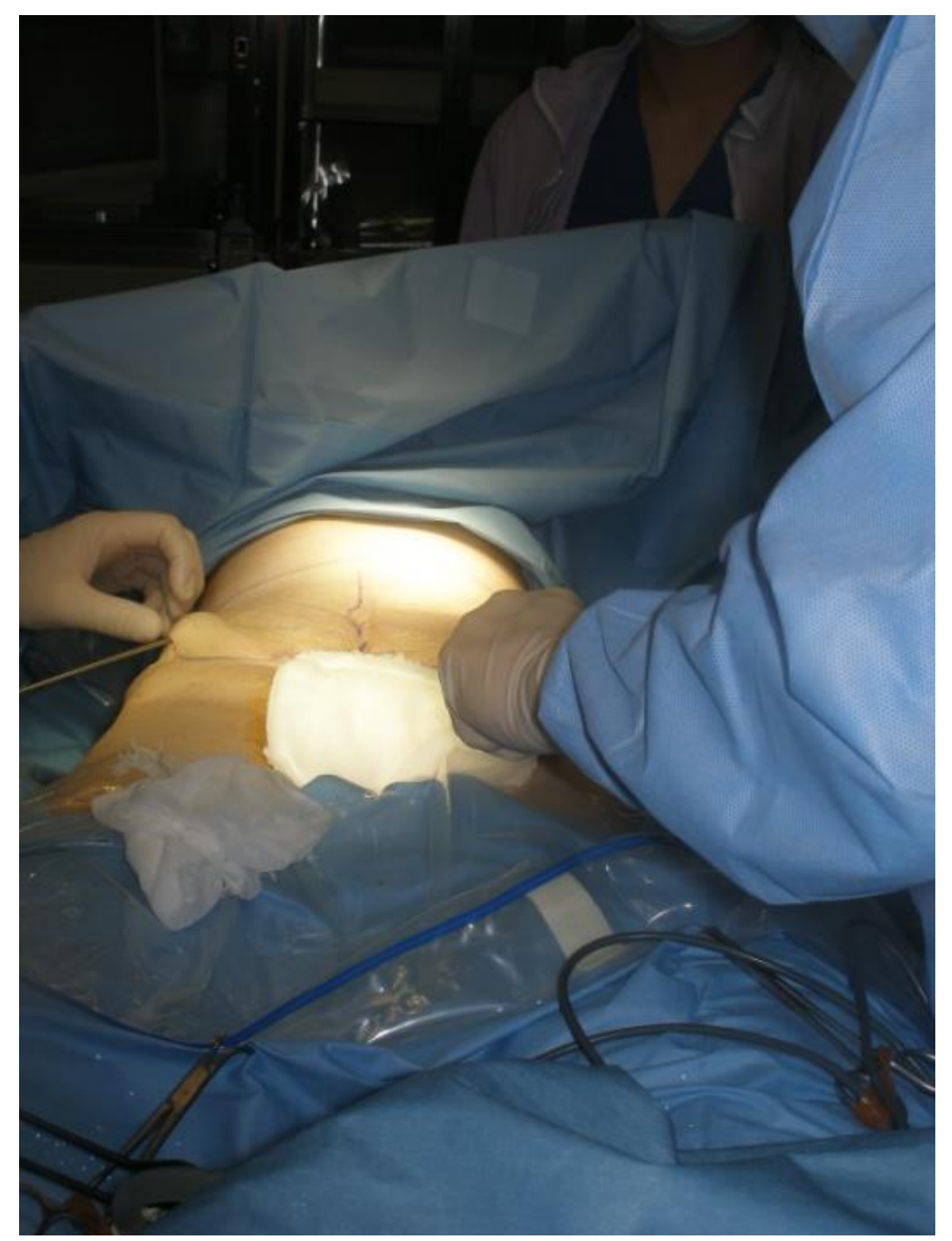


Fig. 1

d

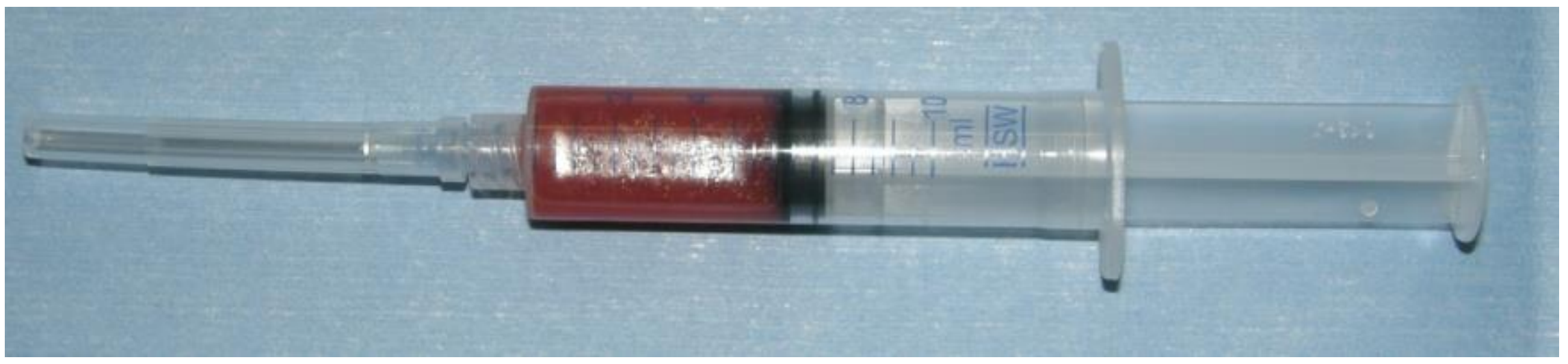


Fig. 3

\section{Therapeutic management of radiation injuries}

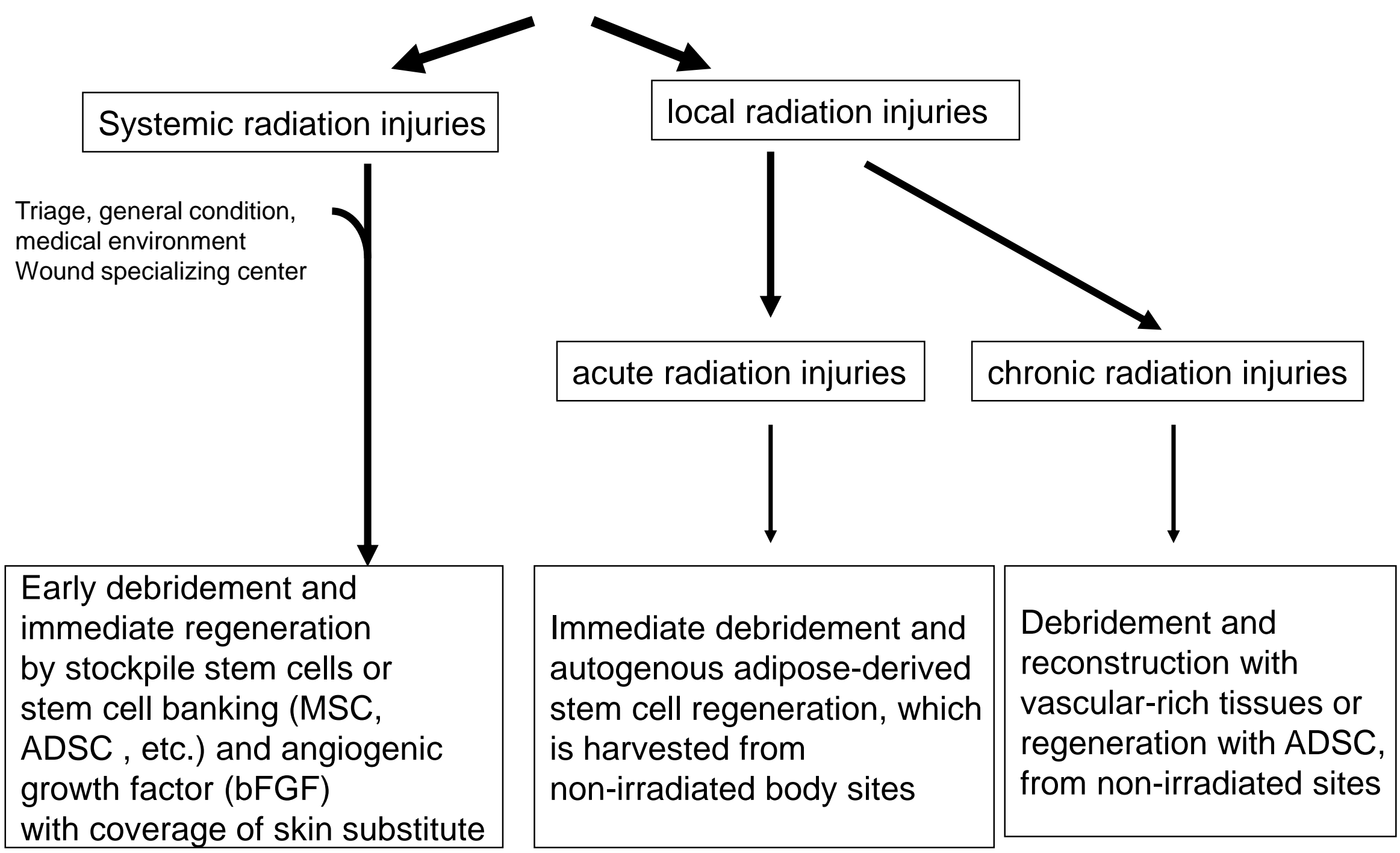

